\title{
Control by arbuscular endomycorrhizae of Pratylenchus brachyurus in pineapple microplants
}

\author{
Jean-Philippe Guillemin, Silvio Gianinazzi, Vivienne Gianinazzi-Pearson \\ and JEAN MARCHAL
}

\begin{abstract}
Guillemin, J.P. ', Gianinazzi, S. ', Gianinazzi-Pearson, V. ' and Marchal, J. ${ }^{2}$ 1994. Control by arbuscular endomycorrhizae of Pratylenchus brachyurus in pineapple microplants. Agricultural Science in Finland 3: 253-262. (' Laboratoire de Phytoparasitologie, INRA-CNRS, Station de Génétique et d'Amélioration des Plantes, INRA, BV 1540, Dijon Cedex, France, and ${ }^{2}$ Laboratoire de Physiologie et Biochimie, CIRAD, FLHOR, Avenue du Val de Montferrant, BP 5035, 34032 Montpellier Cedex 01, France.)
\end{abstract}

\begin{abstract}
Pratylenchus brachyurus (Godfrey) Filip \& Schurr-Steekh. has been reported in association with pineapple roots and is considered as an important pathogen on pineapple. Microplants of Queen Tahiti, Smooth Cayenne and Spanish varieties were inoculated with Glomus sp. (LPA21) and/or P. brachyurus at transplanting from axenic conditions or one month later. The presence of the nematode did not affect shoot growth of endomycorrhizal plants. Late P. brachyurus inoculation did not influence growth of nonmycorrhizal plants while early pathogen application caused reductions in nonmycorrhizal plant growth. Nematode number per $\mathrm{g}$ of root was significantly decreased for endomycorrhizal plants when pathogen was introduced at outplanting or one month later. Nematode inoculation affected endomycorrhizal colonization estimated by non vital staining for the Queen Tahiti and Spanish varieties but did not alter development of metabolically active arbuscules in roots of the three varieties. P concentration of endomycorrhizal shoots was higher for all treatments and $P$. brachyurus tended to decrease mineral concentration of nonmycorrhizal plants with early nematode application.
\end{abstract}

Key words: Ananas comosus, endomycorrhizal infection, vitroplant, pathogen nematode, interactions, integrated control

\section{Introduction}

Arbuscular endomycorrhizal fungi (AMF) and soilborne pathogens occur together in the rhizosphere around plant roots. Arbuscular endomycorrhizae can positively influence plant development by improving mineral nutrition, water uptake, hormone production, resistance to root pathogen or tolerance to pesticides (GIANINAZZI et al. 1982). Guillemin et al. (1991) have shown the benefits to the growth of pineapple microplants of inoculation with endomycorrhizal fungi.
Nematodes are considered an important factor in reducing pineapple production. Pratylenchus brachyurus (Godfrey) Filip \& Schurr-Steekh. causes widespread damage in pineapple plantations, particularly in the Ivory Coast. It was described for the first time in pineapple roots by Godfrey (1929) in Hawaii. This nematode penetrates the elongation zone of roots and develops in the cortical tissue and vascular cylinder (Guérout 1975). Secondary roots can then be destroyed by nematode infestation giving a root system that is essentially composed of primary 
roots (CASWELL et al. 1990). This endopathogen also modifies vegetative plant growth, with reductions in leaf area, and causes a delay in shoot development (LACOeUilHe and Guérout 1976, KeEtch 1982). Decreases in fruit yield can be in the region of 30 to $35 \%$ and the number of suckers may be reduced to $80 \%$ (LACOEUILHE and GuÉROUT 1976, KeETCH 1982). P. brachyurus has a high impact in the Ivory Coast (GUÉROUT 1975), because pineapple is often grown in soil with a $\mathrm{pH}$ adapted to nematode proliferation ( $\mathrm{pH} 5$ to 5.5) (SARAH 1991).

The potential of AMF to alleviate nematodeinduced plant stress has been previously investigated in different plant species but variable hostplant responses to pathogen-endomycorrhiza interactions have been reported (e.g. BAGYARAJ et al. 1979, Kellam and Schenck 1980, Cason et al. 1983, Elliot et al. 1984, COOPER and GRANDISon 1986, Smith 1987, Ingham 1988, Thomas et al. 1989). In the present work, we have tested interactive effects of AMF and P.brachyurus on plant growth, endomycorrhizal infection development and root-colonising nematode populations of micropropagated pineapple.

\section{Material and methods}

Three micropropagated pineapple varieties (Ananas comosus (L.) Merr., Queen Tahiti, Smooth Cayenne (clone CY0) and Spanish varieties) were tested. Plants were raised in a growth chamber under simulated tropical conditions $\left(300 \mu \mathrm{E} \mathrm{s}^{-1}\right.$ $\mathrm{m}^{-2}, 29-25^{\circ} \mathrm{C}, 12 \mathrm{~h}$ day and $70 \%-90 \%$ relative humidity) in a $\gamma$-irradiated (10kGy) acid soil $(\mathrm{pH}$ 5.0). Pineapple microplants were inoculated with root fragments of Tephrosia ehlenbergiana infect- ed with an isolate of Glomus sp. (LPA21) in trays containing a soil:gravel (1:1, v:v) mix during a four-week acclimatization period (M) (GuILLEMIN et al. 1991) or when one month-old weaned microplants were transplanted individually to pots $(1+\mathrm{M})$. Each pot contained $400 \mathrm{~g}$ of the soil:gravel mix and was watered daily with distilled water and weekly with $2 \times 20 \mathrm{ml}$ of Hoagland ${ }^{\circ} 2$ solution (HOAGLAND and ARNON 1950) without phosphate.

Inoculation of Pratylenchus brachyurus was performed with about 100 nematodes per microplant at outplanting from axenic conditions (Nematode) or one month later at the end of the weaning period (Nematode+1).

After 3 months in pots, several growth parameters were evaluated: leaf area $\left(\mathrm{cm}^{2}\right)$, shoot and root fresh mass $(\mathrm{g})$ and shoot dry mass $(\mathrm{g})$ and the $\mathrm{N}, \mathrm{P}, \mathrm{K}, \mathrm{Ca}$ and $\mathrm{Mg}$ concentrations of shoots determined (WARNER and JONES 1967, Comité Inter Instituts pour le diagnostic foliaire 1968, 1972). Endomycorrhiza development was estimated microscopically by the method of TROUVELOT et al. (1986) after clearing roots and staining fungal tissue with trypan blue (PHILIPPS and HAYMAN 1970), or for succinate dehydrogenase (SDH) (SMith and GianinazZi-PEarson 1990) or alkaline phosphatase (ALP) (TISSERANT et al. 1993) activities. Arbuscule frequency (A\%) was also estimated and the proportion of living and functional arbuscules calculated as mentioned below:

$P$. brachyurus in roots was extracted using the non-destructive procedure described by Sarah (1991).

All treatments were tested in the same experiment for Smooth Cayenne and Spanish varieties however two experiments were done for Queen Tahiti variety. Each treatment consisted of 5 rep-

\section{A \% after staining of SDH activity}

Proportion of living arbuscules $=\frac{\mathrm{A} \% \text { after staining with trypan blue }}{\mathrm{A} \%}$

$$
\text { A \% after staining of ALP activity }
$$

Proportion of functional arbuscules $=$

A \% after staining with trypan blue 
licates and statistical analysis of data was performed using Newman-Keuls test following ANOVA.

\section{Results}

Both early (M) and late $(1+\mathrm{M})$ inoculation with the AMF significantly increased the growth of plants compared with nonmycorrhizal controls whether they were infested or not with $P$. brachyurus (Tables 1 and 4).

\section{Endomycorrhiza inoculation at trans- planting from axenic conditions}

With the single exception of Queen Tahiti variety root growth, both shoot and root growth of nonmycorrhizal microplants were significantly reduced when nematodes were introduced at the beginning of the acclimatization period. However, shoot growth of the three pineapple varieties was not significantly modified by nematode infestation in plants inoculated with the Glomus sp. at outplanting from axenic conditions (Table 1). Growth reductions due to the nematode were very limited and only occurred for leaf area (Spanish variety) and roots (Smooth Cayenne and Spanish varieties).

Nematode number per g. of root was significantly lower in endomycorrhizal than nonmycorrhizal roots for all three pineapple varieties (Table 1). Timing of $P$. brachyurus inoculation did not affect nematode numbers developing in the Queen Tahiti and Smooth Cayenne varieties, but later inoculation did reduce pathogen infestation in the Spanish variety (Table 1).

Nematode infestation caused a reduction in $\mathrm{P}$ uptake by nonmycorrhizal plants, particularly for the Smooth Cayenne and Spanish varieties, but not in endomycorrhizal plants (Table 2). For the Spanish variety, $\mathrm{N}, \mathrm{Ca}$ and $\mathrm{Mg}$ concentrations of nonmycorrhizal plants were also decreased by nematode infestation at transplanting. However, in endomycorrhizal plants $\mathrm{P}, \mathrm{Ca}$ and $\mathrm{Mg}$ concentrations in shoots of the three varieties inoculated with the nematodes were comparable to those of endomycorrhizal plants growing in the absence of nematodes (Table 2).

Nematode application at outplanting significantly reduced arbuscule frequency (A\%) for the Queen Tahiti and Spanish varieties after trypan blue staining (Table 3 ) but this reduction disappeared with pathogen inoculation one month later. This showed that it was of greatest benefit to establish endomycorrhizal colonization as soon as possible. Reduction of $\mathrm{A} \%$ was also observed after staining for SDH and ALP activities in the Queen Tahiti variety (Table 3). The proportion of living and functional arbuscules was not altered by nematode infestation (Table 3 ).

\section{Endomycorrhizal inoculation at outplanting to pots}

Growth of endomycorrhizal plants was significantly greater than of nonmycorrhizal plants (Table 4). Inoculation with $P$. brachyurus at outplanting reduced the growth of nonmycorrhizal but not endomycorrhizal plants (Table 4).

The nematode population was reduced in endomycorrhizal plants with both timings of the pathogen application for the Queen Tahiti variety (Table 4) but this reduction was observed for the Smooth Cayenne and Spanish varieties only when nematodes and endomycorrhizae were inoculated simultaneously. Endomycorrhiza development after nematode application did not influence nematode infestation of roots of the Smooth Cayenne and Spanish varieties. Timing of pathogen application influenced its presence in the roots of the Spanish variety; indeed the population was greater when the pathogen was introduced before inoculation with the AMF.

Nematodes negatively affected $\mathrm{P}$ uptake by nonmycorrhizal but not endomycorrhizal plants (Table 5). Endomycorrhiza formation enhanced $\mathrm{Ca}$ and $\mathrm{Mg}$ contents of the Queen Tahiti and Spanish varieties and $\mathrm{N}$ concentration for the Spanish variety. $\mathrm{K}$ contents were higher for the nonmycorrhizal plants.

P. brachyurus application at outplanting sig- 
Table 1: Leaf area (LA), shoot (SFM) and root (RFM) fresh mass, shoot dry mass (SDM) and number of nematodes per g. of roots (Nem root) of nonmycorrhizal (NM) and endomycorrhizal pineapple at transplanting from axenic conditions (M): nematode uninoculated (Control), inoculated (Nematode) and inoculated one month later (Nematode +1 ).

A - Queen Tahiti variety

\begin{tabular}{llrrrrr}
\hline & & LA $\left(\mathrm{cm}^{2}\right)$ & SFM $(\mathrm{g})$ & RFM $(\mathrm{g})$ & SDM $(\mathrm{g})$ & Nem. root \\
\hline Control & $\mathrm{NM}$ & $186,9 \mathrm{~b}$ & $15.26 \mathrm{~b}$ & $1.76 \mathrm{~b}$ & $1.54 \mathrm{~b}$ & $0 \mathrm{c}$ \\
& $\mathrm{M}$ & $460.7 \mathrm{a}$ & $36.01 \mathrm{a}$ & $4.00 \mathrm{a}$ & $3.70 \mathrm{a}$ & $0 \mathrm{c}$ \\
\multirow{2}{*}{ Nematode } & $\mathrm{NM}$ & $100.5 \mathrm{c}$ & $7.35 \mathrm{c}$ & $1.25 \mathrm{~b}$ & $0.91 \mathrm{~b}$ & $396 \mathrm{a}$ \\
& $\mathrm{M}$ & $353.7 \mathrm{ab}$ & $27.31 \mathrm{ab}$ & $3.12 \mathrm{a}$ & $3.16 \mathrm{a}$ & $232 \mathrm{~b}$ \\
\multirow{2}{*}{ Nematode+1 } & $\mathrm{NM}$ & $209.0 \mathrm{~b}$ & $13.58 \mathrm{~b}$ & $1.71 \mathrm{~b}$ & $1.51 \mathrm{~b}$ & $330 \mathrm{a}$ \\
& $\mathrm{M}$ & $444.4 \mathrm{a}$ & $34.16 \mathrm{a}$ & $3.96 \mathrm{a}$ & $3.58 \mathrm{a}$ & $250 \mathrm{~b}$ \\
\hline
\end{tabular}

B - Smooth Cayenne variety

\begin{tabular}{llrrrrr}
\hline & & LA $\left(\mathrm{cm}^{2}\right)$ & SFM $(\mathrm{g})$ & RFM $(\mathrm{g})$ & SDM $(\mathrm{g})$ & Nem. root \\
\hline \multirow{2}{*}{ Control } & $\mathrm{NM}$ & $299.8 \mathrm{c}$ & $23.35 \mathrm{c}$ & $2.63 \mathrm{c}$ & $2.08 \mathrm{~b}$ & $0 \mathrm{c}$ \\
& $\mathrm{M}$ & $640.9 \mathrm{a}$ & $54.87 \mathrm{a}$ & $5.48 \mathrm{a}$ & $5.02 \mathrm{a}$ & $0 \mathrm{c}$ \\
\multirow{2}{*}{ Nematode } & $\mathrm{NM}$ & $178.6 \mathrm{~d}$ & $13.82 \mathrm{~d}$ & $1.64 \mathrm{~d}$ & $1.39 \mathrm{c}$ & $456 \mathrm{a}$ \\
& $\mathrm{M}$ & $540.6 \mathrm{ab}$ & $42.86 \mathrm{ab}$ & $3.44 \mathrm{~b}$ & $4.58 \mathrm{a}$ & $267 \mathrm{~b}$ \\
\multirow{2}{*}{ Nematode+1 } & $\mathrm{NM}$ & $232.7 \mathrm{~cd}$ & $19.75 \mathrm{c}$ & $2.11 \mathrm{c}$ & $2.09 \mathrm{~b}$ & $418 \mathrm{a}$ \\
& $\mathrm{M}$ & $547.5 \mathrm{ab}$ & $48.38 \mathrm{a}$ & $4.06 \mathrm{~b}$ & $4.64 \mathrm{a}$ & $212 \mathrm{~b}$ \\
\hline
\end{tabular}

C - Spanish variety

\begin{tabular}{llrrrrr}
\hline & & LA $\left(\mathrm{cm}^{2}\right)$ & SFM $(\mathrm{g})$ & RFM $(\mathrm{g})$ & SDM $(\mathrm{g})$ & Nem. root \\
\hline \multirow{2}{*}{ Control } & $\mathrm{NM}$ & $313.2 \mathrm{c}$ & $21.70 \mathrm{~b}$ & $2.05 \mathrm{c}$ & $2.25 \mathrm{c}$ & $0 \mathrm{~d}$ \\
& $\mathrm{M}$ & $537.1 \mathrm{a}$ & $38.48 \mathrm{a}$ & $3.97 \mathrm{a}$ & $3.91 \mathrm{a}$ & $0 \mathrm{~d}$ \\
\multirow{2}{*}{ Nematode } & $\mathrm{NM}$ & $96.4 \mathrm{~d}$ & $5.72 \mathrm{c}$ & $0.75 \mathrm{~d}$ & $0.74 \mathrm{~d}$ & $350 \mathrm{a}$ \\
& $\mathrm{M}$ & $476.9 \mathrm{~b}$ & $34.47 \mathrm{a}$ & $2.77 \mathrm{~b}$ & $3.40 \mathrm{ab}$ & $157 \mathrm{~b}$ \\
\multirow{2}{*}{ Nematode +1} & $\mathrm{NM}$ & $263.1 \mathrm{c}$ & $19.57 \mathrm{~b}$ & $2.26 \mathrm{c}$ & $1.97 \mathrm{c}$ & $180 \mathrm{~b}$ \\
& $\mathrm{M}$ & $543.6 \mathrm{a}$ & $39.83 \mathrm{a}$ & $3.40 \mathrm{a}$ & $4.04 \mathrm{a}$ & $46 \mathrm{c}$ \\
\hline
\end{tabular}

Values in a column followed by different letters are significantly different $(\mathrm{p}<0.05)$

nificantly reduced arbuscule frequency (A\%) determined after trypan blue staining in the Queen Tahiti and Spanish varieties (Table 3). A\% evaluated by SDH and ALP activities was negatively affected by nematodes for the Queen Tahiti variety. However, the proportion of living and functional arbuscules was not modified by $P$. brachyurus for the three pineapple varieties (Table 3).

\section{Discussion}

Although $P$. brachyurus reduced the growth of nonmycorrhizal plants in all three pineapple varieties, in those colonized by AMF growth was not significantly affected. Precolonization of roots by AMF can therefore reduce the harmful effects of nematodes on plant growth. Simultaneous symbiont and pathogen inoculation at transplanting to pots did not affect the growth of endomycorrhizal pineapple. However, simultaneous inoculation of the AMF and nematodes at outplanting from axenic conditions slightly reduced growth of endomycorrhizal plants of the Queen Tahiti and Smooth Cayenne varieties. Both micro-organisms can be an important photosynthate sink for very young micropropagated plantlets. Effects of the nematode on young microplants of pineapple during the acclimatization period are not irreversible; indeed, late endomycorrhizal coloniza- 
Table 2: Mineral concentration (\% of dry mass) of shoots of nonmycorrhizal (NM) and endomycorrhizal pineapple at outplanting from axenic conditions (M): nematode uninoculated (Control), inoculated (Nematode) and inoculated one month later (Nematode +1$)$.

A - Queen Tahiti variety

\begin{tabular}{|c|c|c|c|c|c|c|}
\hline & & $\mathrm{N}$ & $\mathrm{P}$ & $\mathrm{K}$ & $\mathrm{Ca}$ & $\mathrm{Mg}$ \\
\hline \multirow[t]{2}{*}{ Control } & $\mathrm{NM}$ & 2.04 & 0.08 & 4.60 & 0.81 & 0.29 \\
\hline & M & 1.70 & 0.18 & 3.59 & 0.96 & 0.37 \\
\hline \multirow[t]{2}{*}{ Nematode } & $\mathrm{NM}$ & 1.65 & 0.08 & 3.64 & 0.82 & 0.28 \\
\hline & M & 1.87 & 0.15 & 3.77 & 1.00 & 0.37 \\
\hline \multirow[t]{2}{*}{ Nematode +1} & NM & 1.83 & 0.05 & 4.01 & 0.87 & 0.28 \\
\hline & M & 1.77 & 0.18 & 3.49 & 1.04 & 0.37 \\
\hline
\end{tabular}

B - Smooth Cayenne variety

\begin{tabular}{lllllll}
\hline & & $\mathrm{N}$ & $\mathrm{P}$ & $\mathrm{K}$ & $\mathrm{Ca}$ & $\mathrm{Mg}$ \\
\hline \multirow{2}{*}{ Control } & $\mathrm{NM}$ & 2.03 & 0.13 & 4.46 & 1.05 & 0.35 \\
& $\mathrm{M}$ & 1.99 & 0.13 & 4.07 & 1.17 & 0.34 \\
Nematode & $\mathrm{NM}$ & 1.93 & 0.07 & 4.52 & 1.08 & 0.35 \\
& $\mathrm{M}$ & 1.81 & 0.14 & 3.54 & 1.09 & 0.32 \\
\multirow{2}{*}{ Nematode+1 } & $\mathrm{NM}$ & 2.09 & 0.09 & 4.90 & 1.11 & 0.30 \\
& $\mathrm{M}$ & 1.76 & 0.14 & 3.62 & 1.22 & 0.34 \\
\hline
\end{tabular}

C - Spanish variety

\begin{tabular}{lllllll}
\hline & & $\mathrm{N}$ & $\mathrm{P}$ & $\mathrm{K}$ & $\mathrm{Ca}$ & $\mathrm{Mg}$ \\
\hline \multirow{2}{*}{ Control } & $\mathrm{NM}$ & 1.88 & 0.13 & 4.13 & 0.75 & 0.26 \\
& $\mathrm{M}$ & 1.63 & 0.12 & 3.30 & 0.82 & 0.28 \\
Nematode & $\mathrm{NM}$ & 1.41 & 0.04 & 3.67 & 0.60 & 0.19 \\
& $\mathrm{M}$ & 1.67 & 0.14 & 3.06 & 0.95 & 0.30 \\
\multirow{2}{*}{ Nematode+1 } & $\mathrm{NM}$ & 1.82 & 0.09 & 4.06 & 0.89 & 0.26 \\
& $\mathrm{M}$ & 1.64 & 0.14 & 3.01 & 0.90 & 0.30 \\
\hline
\end{tabular}

tion at transplanting to pots can compensate growth reductions of plants inoculated with nematodes at outplanting from axenic conditions. $\mathrm{Mi}$ cropropagated plantlets could tolerate better the nematode inoculation at outplanting from axenic conditions followed one month later by endomycorrhizal inoculation than both symbiotic and pathogen inoculations at the beginning of the acclimatization period. The application of nematodes at outplanting from axenic conditions without endomycorrhizal inoculation significantly reduced plant growth but this effect was not observed when plants were infested by nematodes one month later. This supports previous observations that older plants can tolerate pathogen infestation better than younger plants (COOPER and GRANDISON 1986).

Pathogen effects on endomycorrhizal colonization estimated after non vital staining varied with the pineapple variety. When nematodes were applied at transplanting this significantly reduced arbuscule frequency (A\%) in the Queen Tahiti and Spanish varieties. The ability of nematodes to reduce endomycorrhizal development has also been observed by several authors (e.g. O'BANNON and Nemec 1979, Elliot et al. 1984), and it has been suggested that nematodes could induce an unfavourable environment for infection by the fungal symbiont (Tномas et al. 1989). Although nematode infestation negatively influenced val- 
Table 3: Arbuscular frequency observed after staining with trypan blue (TB), succinate dehydrogenase (SDH) or alkaline phosphatase activities and proportion of living (SDH/TB) and functional (ALP/TB) arbuscules of endomycorrhizal pineapple roots at outplanting from axenic conditions $(\mathrm{M})$ and at transplanting to pots $(1+\mathrm{M})$ : nematode uninoculated (Control), inoculated (Nematode) and inoculated one month later (Nematode +1$)$.

\begin{tabular}{|c|c|c|c|c|c|c|}
\hline & & \multicolumn{5}{|c|}{ Frequency of arbuscules (A\%) detected by staining for } \\
\hline & & $\begin{array}{l}\text { Total fungal } \\
\text { tissue (TB) }\end{array}$ & $\begin{array}{c}\text { SDH } \\
\text { activity (\%) }\end{array}$ & $\mathrm{SDH} / \mathrm{TB}$ & $\begin{array}{c}\text { ALB } \\
\text { activity (\%) }\end{array}$ & ALB/TB \\
\hline \multicolumn{7}{|c|}{ Queen Tahiti variety } \\
\hline \multirow[t]{3}{*}{ M } & Control & $63 \mathrm{a}$ & $24.5 \mathrm{a}$ & $0.39 \mathrm{a}$ & $16.4 \mathrm{a}$ & $0.26 \mathrm{a}$ \\
\hline & Nematode & $46 \mathrm{~b}$ & $14.7 \mathrm{~b}$ & $0.32 \mathrm{a}$ & $9.4 \mathrm{~b}$ & $0.21 \mathrm{a}$ \\
\hline & Nematode+1 & $56 a b$ & $17.4 \mathrm{~b}$ & $0.31 \mathrm{a}$ & $12.0 \mathrm{~b}$ & $0.22 \mathrm{a}$ \\
\hline \multirow[t]{3}{*}{$1+\mathrm{M}$} & Control & $65 \mathrm{a}$ & $19.0 \mathrm{a}$ & $0.29 \mathrm{a}$ & $12.7 \mathrm{a}$ & $0.20 \mathrm{a}$ \\
\hline & Nematode & $33 \mathrm{~b}$ & $12.7 \mathrm{~b}$ & $0.38 \mathrm{a}$ & $8.0 \mathrm{~b}$ & $0.24 \mathrm{a}$ \\
\hline & Nematode +1 & $38 \mathrm{~b}$ & $13.6 \mathrm{~b}$ & $0.36 \mathrm{a}$ & $9.2 \mathrm{~b}$ & $0.24 \mathrm{a}$ \\
\hline \multicolumn{7}{|c|}{ Smooth Cayenne variety } \\
\hline \multirow[t]{3}{*}{ M } & Control & $65 \mathrm{a}$ & $20.3 \mathrm{a}$ & $0.31 \mathrm{a}$ & $15.8 \mathrm{a}$ & $0.24 \mathrm{a}$ \\
\hline & Nematode & $50 \mathrm{a}$ & $20.0 \mathrm{a}$ & $0.40 \mathrm{a}$ & $13.9 \mathrm{a}$ & $0.27 \mathrm{a}$ \\
\hline & Nematode +1 & $51 \mathrm{a}$ & $17.5 \mathrm{a}$ & $0.34 \mathrm{a}$ & $14.0 \mathrm{a}$ & $0.27 \mathrm{a}$ \\
\hline \multirow[t]{3}{*}{$1+\mathrm{M}$} & Control & $53 \mathrm{a}$ & $13.8 \mathrm{a}$ & $0.26 \mathrm{a}$ & $11.0 \mathrm{a}$ & $0.21 \mathrm{a}$ \\
\hline & Nematode & $60 \mathrm{a}$ & $10.9 \mathrm{a}$ & $0.18 \mathrm{a}$ & $8.0 \mathrm{a}$ & $0.13 \mathrm{a}$ \\
\hline & Nematode +1 & $68 \mathrm{a}$ & $14.9 \mathrm{a}$ & $0.22 \mathrm{a}$ & $10.3 \mathrm{a}$ & $0.15 \mathrm{a}$ \\
\hline \multicolumn{7}{|c|}{ Spanish variety } \\
\hline \multirow[t]{3}{*}{ M } & Control & $50 \mathrm{a}$ & $21.6 \mathrm{a}$ & $0.43 \mathrm{a}$ & $13.8 \mathrm{a}$ & $0.28 \mathrm{a}$ \\
\hline & Nematode & $29 \mathrm{~b}$ & $16.5 \mathrm{a}$ & $0.56 \mathrm{a}$ & $10.2 \mathrm{a}$ & $0.35 \mathrm{a}$ \\
\hline & Nematode+1 & $38 \mathrm{ab}$ & $18.6 \mathrm{a}$ & $0.49 \mathrm{a}$ & $10.0 \mathrm{a}$ & $0.27 \mathrm{a}$ \\
\hline \multirow{3}{*}{$1+\mathrm{M}$} & Control & $40 \mathrm{a}$ & $18.6 \mathrm{a}$ & $0.46 a$ & $12.2 \mathrm{a}$ & $0.30 \mathrm{a}$ \\
\hline & Nematode & $31 \mathrm{~b}$ & $16.7 \mathrm{a}$ & $0.54 \mathrm{a}$ & $9.4 \mathrm{a}$ & $0.30 \mathrm{a}$ \\
\hline & Nematode +1 & $35 \mathrm{ab}$ & $14.4 \mathrm{a}$ & $0.41 \mathrm{a}$ & $9.4 \mathrm{a}$ & $0.27 \mathrm{a}$ \\
\hline
\end{tabular}

Values for each combination of variety and inoculation treatments (nematode and endomycorrhizal fungus) followed by different letters are significantly different $(\mathrm{p}<0.05)$

ues for A\% of Queen Tahiti variety estimated by SDH and ALP activities, P. brachyurus did not affect the proportion of living and functional arbuscules of the three pineapple varieties, and consequently did not influence the efficiency of the symbiosis for pineapple. This could partly explain the lack of effect of $P$. brachyurus on the growth of endomycorrhizal plants.

Several reports have shown that endomycorrhizal colonization decreases nematode populations in root systems (e.g. BAGYARAJ et al. 1979, SAleH and SiKora 1984), and Smith et al. (1986) showed that AMF can enhance plant tolerance to nematodes in field conditions. In this study, numbers of nematodes were also significantly reduced in the roots of endomycorrhizal pineapple of the three varieties in comparison to nonmycorrhizal plants, whether nematodes were applied simultaneously with or after the AMF. Reductions in nematode infection have been attributed to modifications in plant physiology caused by the symbiotic fungi. AMF are able to ensure an adequate $\mathrm{P}$ nutrition in presence of nematodes and since $\mathrm{P}$ is considered as an important factor in plant tolerance (SMITH and KAPLAN 1988), higher concentrations of this element in endomycorrhizal tissues could have a direct action reducing nematode numbers in roots (MACGuIDwIN et al. 1985). Changes in root exudates may also alter root attractiveness for nematodes (MACGUIDwIN et al. 1985), or induce physical and chemical barriers to root penetration (KELLAM and SCHENCK 1980). 
Table 4: Leaf area (LA), shoot (SFM) and root (RFM) fresh mass, shoot dry mass (SDM) and number of nematodes per g. of roots (Nem root) of nonmycorrhizal (NM) and endomycorrhizal pineapple at transplanting to pot $(1+\mathrm{M})$ : nematode uninoculated (Control), inoculated (Nematode) and inoculated one month later (Nematode+1).

A - Queen Tahiti variety

\begin{tabular}{llrrrrr}
\hline & & LA $\left(\mathrm{cm}^{2}\right)$ & SFM $(\mathrm{g})$ & RFM $(\mathrm{g})$ & SDM $(\mathrm{g})$ & Nem. root \\
\hline Control & $\mathrm{NM}$ & $312.4 \mathrm{~b}$ & $24.82 \mathrm{~b}$ & $2.52 \mathrm{~b}$ & $2.58 \mathrm{~b}$ & $0 \mathrm{c}$ \\
& $1+\mathrm{M}$ & $452.9 \mathrm{a}$ & $33.85 \mathrm{a}$ & $3.19 \mathrm{a}$ & $3.36 \mathrm{a}$ & $0 \mathrm{c}$ \\
\multirow{2}{*}{ Nematode } & $\mathrm{NM}$ & $136.9 \mathrm{c}$ & $10.95 \mathrm{c}$ & $1.19 \mathrm{c}$ & $1.13 \mathrm{c}$ & $564 \mathrm{a}$ \\
& $1+\mathrm{M}$ & $389.5 \mathrm{ab}$ & $31.62 \mathrm{a}$ & $2.74 \mathrm{ab}$ & $3.00 \mathrm{a}$ & $300 \mathrm{~b}$ \\
\multirow{2}{*}{ Nematode+1 } & $\mathrm{NM}$ & $176.7 \mathrm{c}$ & $13.11 \mathrm{c}$ & $1.26 \mathrm{c}$ & $1.28 \mathrm{c}$ & $465 \mathrm{a}$ \\
& $1+\mathrm{M}$ & $433.8 \mathrm{a}$ & $35.26 \mathrm{a}$ & $2.77 \mathrm{ab}$ & $3.53 \mathrm{a}$ & $304 \mathrm{~b}$ \\
\hline
\end{tabular}

B - Smooth Cayenne variety

\begin{tabular}{llrrrrr}
\hline & & LA $\left(\mathrm{cm}^{2}\right)$ & SFM $(\mathrm{g})$ & RFM $(\mathrm{g})$ & SDM $(\mathrm{g})$ & Nem. root \\
\hline Control & $\mathrm{NM}$ & $299.8 \mathrm{~b}$ & $23.35 \mathrm{~b}$ & $2.63 \mathrm{~b}$ & $2.08 \mathrm{~b}$ & $0 \mathrm{c}$ \\
& $1+\mathrm{M}$ & $471.7 \mathrm{a}$ & $34.65 \mathrm{a}$ & $3.06 \mathrm{a}$ & $3.07 \mathrm{a}$ & $0 \mathrm{c}$ \\
\multirow{2}{*}{ Nematode } & $\mathrm{NM}$ & $178.6 \mathrm{c}$ & $13.82 \mathrm{c}$ & $1.64 \mathrm{c}$ & $1.39 \mathrm{c}$ & $456 \mathrm{a}$ \\
& $1+\mathrm{M}$ & $362.3 \mathrm{ab}$ & $31.24 \mathrm{a}$ & $2.84 \mathrm{a}$ & $3.65 \mathrm{a}$ & $389 \mathrm{a}$ \\
\multirow{2}{*}{ Nematode+1 } & $\mathrm{NM}$ & $232.7 \mathrm{bc}$ & $19.75 \mathrm{~b}$ & $2.11 \mathrm{~b}$ & $2.09 \mathrm{~b}$ & $418 \mathrm{a}$ \\
& $1+\mathrm{M}$ & $485.1 \mathrm{a}$ & $35.47 \mathrm{a}$ & $3.05 \mathrm{a}$ & $3.85 \mathrm{a}$ & $293 \mathrm{~b}$ \\
\hline
\end{tabular}

C - Spanish variety

\begin{tabular}{llrrrrr}
\hline & & LA $\left(\mathrm{cm}^{2}\right)$ & SFM $(\mathrm{g})$ & RFM $(\mathrm{g})$ & SDM $(\mathrm{g})$ & Nem. root \\
\hline Control & $\mathrm{NM}$ & $313.2 \mathrm{~b}$ & $21.70 \mathrm{~b}$ & $2.05 \mathrm{~b}$ & $2.25 \mathrm{~b}$ & $0 \mathrm{~d}$ \\
& $1+\mathrm{M}$ & $483.5 \mathrm{a}$ & $33.45 \mathrm{a}$ & $2.92 \mathrm{a}$ & $3.23 \mathrm{a}$ & $0 \mathrm{~d}$ \\
\multirow{2}{*}{ Nematode } & $\mathrm{NM}$ & $96.4 \mathrm{c}$ & $5.72 \mathrm{c}$ & $0.75 \mathrm{c}$ & $0.74 \mathrm{c}$ & $350 \mathrm{a}$ \\
& $1+\mathrm{M}$ & $465.0 \mathrm{a}$ & $33.68 \mathrm{a}$ & $2.29 \mathrm{ab}$ & $3.40 \mathrm{a}$ & $330 \mathrm{a}$ \\
\multirow{2}{*}{ Nematode+1 } & $\mathrm{NM}$ & $263.1 \mathrm{~b}$ & $19.57 \mathrm{~b}$ & $2.26 \mathrm{ab}$ & $1.97 \mathrm{~b}$ & $180 \mathrm{~b}$ \\
& $1+\mathrm{M}$ & $432.7 \mathrm{a}$ & $31.29 \mathrm{a}$ & $2.65 \mathrm{a}$ & $2.85 \mathrm{a}$ & $122 \mathrm{c}$ \\
\hline
\end{tabular}

Values in a column followed by different letters are significantly different $(\mathrm{p}<0.05)$

Endomycorrhizal colonization could also represent a competition for photosynthates in roots (SMITH 1987), and thus produce a less favourable environment for the nematodes (Kellam and SCHENCK 1980) or influence the quality of food reserves of nematodes (MACGUIDWIN et al. 1985). Endomycorrhizal plants have higher sugar contents, modified hormone balance and modifications in the composition of amino acids (e.g increases in serine and phenylalanine which are nematicidal) (SURESH et al. 1985). Presence of a fungal symbiont in roots can affect the normal life cycle of nematodes (CASON et al. 1983) and reduce nematode size (Sitaramaiah and Sikora 1982).
Other micro-organisms such as bacteria and fungi are also considered as antagonists to nematodes (CAYrol et al. 1992), and the combination of AMF with one or several antagonists could produce a more beneficial synergistic action on plant protection and growth. Fallow could be also used to combat nematode populations in soil (STIRLING and NIKULIN 1993), but this approach risks decreasing endomycorrhizal potential and reducing soil fertility (SARAH 1987) unless inoculation with efficient AMF after fallow is ensured. The control of nematodes in pineapple, which avoids excess use of nematicides, clearly requires an integrated approach. The results reported here suggest that endomycorrhizae, which are not affect- 
Table 5: Mineral concentration (\% of dry mass) of shoots of nonmycorrhizal (NM) and endomycorrhizal pineapple at transplanting to pots $(1+\mathrm{M})$ : nematode uninoculated (Control), inoculated (Nematode) and inoculated one month later (Nematode+1).

A - Queen Tahiti variety

\begin{tabular}{lllllll}
\hline & & $\mathrm{N}$ & $\mathrm{P}$ & $\mathrm{K}$ & $\mathrm{Ca}$ & $\mathrm{Mg}$ \\
\hline Control & $\mathrm{NM}$ & 1.72 & 0.10 & 3.83 & 0.83 & 0.33 \\
& $\mathrm{H}+\mathrm{M}$ & 1.76 & 0.17 & 3.74 & 0.79 & 0.33 \\
Nematode & $\mathrm{NM}$ & 1.83 & 0.05 & 4.60 & 0.67 & 0.24 \\
& $1+\mathrm{M}$ & 1.95 & 0.13 & 3.91 & 0.88 & 0.34 \\
Nematode+1 & $\mathrm{NM}$ & 2.22 & 0.08 & 5.25 & 0.74 & 0.27 \\
& $1+\mathrm{M}$ & 1.81 & 0.12 & 3.42 & 0.91 & 0.32 \\
\hline
\end{tabular}

B - Smooth Cayenne variety

\begin{tabular}{lllllll}
\hline & & $\mathrm{N}$ & $\mathrm{P}$ & $\mathrm{K}$ & $\mathrm{Ca}$ & $\mathrm{Mg}$ \\
\hline Control & $\mathrm{NM}$ & 2.03 & 0.13 & 4.46 & 1.05 & 0.35 \\
& $1+\mathrm{M}$ & 2.26 & 0.16 & 4.46 & 1.18 & 0.33 \\
Nematode & $\mathrm{NM}$ & 1.93 & 0.07 & 4.52 & 1.08 & 0.35 \\
& $1+\mathrm{M}$ & 1.70 & 0.16 & 3.38 & 1.03 & 0.33 \\
Nematode +1 & $\mathrm{NM}$ & 2.09 & 0.09 & 4.90 & 1.11 & 0.30 \\
& $1+\mathrm{M}$ & 1.85 & 0.16 & 3.68 & 1.13 & 0.35 \\
\hline
\end{tabular}

C - Spanish variety

\begin{tabular}{lllllll}
\hline & & $\mathrm{N}$ & $\mathrm{P}$ & $\mathrm{K}$ & $\mathrm{Ca}$ & $\mathrm{Mg}$ \\
\hline Control & $\mathrm{NM}$ & 1.88 & 0.13 & 4.13 & 0.75 & 0.26 \\
& $\mathrm{I}+\mathrm{M}$ & 1.97 & 0.16 & 4.01 & 0.83 & 0.27 \\
Nematode & $\mathrm{NM}$ & 1.41 & 0.04 & 3.67 & 0.60 & 0.19 \\
& $1+\mathrm{M}$ & 1.74 & 0.13 & 3.18 & 0.83 & 0.26 \\
Nematode+1 & $\mathrm{NM}$ & 1.82 & 0.09 & 4.06 & 0.89 & 0.26 \\
& $1+\mathrm{M}$ & 1.98 & 0.14 & 3.98 & 0.91 & 0.27 \\
\hline
\end{tabular}

ed by nematicides (HABTE and MANJUNATH 1988), could be a valuable component in a scheme of integrated protection against nematodes.
Acknowledgements. The authors thank Vitropic S.A. (Montpellier, France) for supplying the micropropagated plant material and Dr J.L. Sarah (CIRAD, FHLOR, Montpellier, France) for supplying P. brachyurus.

\section{References}

Bagyaraj, D.J., Manjunath, A. \& RedDy, D.D.R. 1979. Interaction of vesicular-arbuscular mycorrhiza fungi with root-knot nematodes in tomato. Plant and Soil 51: 397-403.

CASON, K.M.T., HuSSEY, R.S. \& RONCADORI, R.W. 1983. Interaction of vesicular-arbuscular mycorrhizal fungi and phosphorus with Meloidogyne incognita on tomato. Journal of Nematology 15: 410-417.

Caswell, E.P., Sarah, J.L. \& APT, W.J. 1990. Nematode parasites of pineapple. In: Luc, M., Sikora, R.A. \&
Bridge, J. (eds.). Plant parasitic nematodes in subtropical and tropical agriculture. CAB International. p. 519-537.

Cayrol, J.C., Ditan-Caporalion, C. \& Panchaud-MatTEI, E. 1992. La lutte biologique contre les nématodes phytoparasites. Courrier de la Cellule Environnement de I'INRA 17: 31-44.

Comité Inter Instituts pour le diagnostic foliaire 1968. Méthode de référence pour la détermination des éléments minéraux dans les végétaux. Coll. Eur. Méd. sur 
le contrôle de l'alimentation des Plantes cultivées, Séville. p. 12-20.

- 1972. Méthode de référence pour la détermination des éléments minéraux dans les végétaux. Coll. Eur. Méd. sur le contrôle de l'alimentation des Plantes cultivées, Budapest. p. 144-150.

COOPER, K.M. \& Grandison, G.S. 1986. Interaction of vesicular-arbuscular mycorrhizal fungi and root-knot nematode on cultivars of tomato and white clover susceptible to Meloidogyne hapla. Annals of Applied Biology 108: 555-565.

ElLIOT, A.P., BIRD, G.W. \& SAFIR, G.R. 1984. Joint influence of Pratylenchus penetrans (nematoda) and Glomus fasciculatum (phytomyceta) on the ontogeny of Phaseolus vulgaris. Nematropica 14: 111-119.

Gianinazzi, S., Gianinazzi-Pearson, V. \& Trouvelot, A. 1982. Les Mycorhizes, Partie Intégrante de la Plante: Biologie et Perspective d'Utilisation. 397 p. INRAPresse, Paris, France.

GODFREY, G.H. 1929. A destructive root disease of pineapple and other plants due to Tylenchus brachyurus. Phytopathology 19: 611-629.

GUÉrout, R. 1975. Nematodes of pineapple: a review. Pest Articles \& News Summaries 21: 123-140.

Guillemin, J.P., Gianinazzi, S. \& Gianinazzi-Pearson, V. 1991. L'endomycorhization de vitroplants d'Ananas comosus: mise en évidence d'un effet mycorhizien. Fruits 46: 355-358.

HabTe, M. \& Manjunath, A. 1988. Influence of phenamiphos on the vesicular-arbuscular mycorrhizal symbiosis in Leucaena leucocephala. Biology and Fertility of Soils 5: 313-316.

Hoagland, D.R. \& ARNON, D.I. 1950. The water-culture method for growing plants without soil. California Agricultural Experiment Station Circular 347: 1-32.

INGHAM, R.E. 1988. Interactions between nematodes and vesicular-arbuscular mycorrhizae. Agriculture, Ecosystems and Environment 24: 169-182.

KEETCH, D.P. 1982. Nematode pests of pineapple. In: Keetch, D.P. \& Heyns, J. (eds.). Nematology in South Africa. Department of Agriculture and Fisheries, Pretoria. p. 19-29.

Kellam, M.K. \& SChENCK, N.C. 1980. Interactions between a vesicular-arbuscular mycorrhizal fungus and root-knot nematode on soybean. Phytopathology 70 : 293-296.

LACOEUILhe, J.J. \& Guérout, R. 1976. Action du nématode Pratylenchus brachyurus sur la croissance, la nutrition et les rendements de l'ananas 'Cayenne lisse'. Influence de la localisation de la fumure. Fruits 31 : 147-156.

MACGuidwin, A.E., BIRD, G.W. \& SAFIR, G.R. 1985. Influence of Glomus fasciculatum on Meloidogyne hapla infecting Allium cepa. Journal of Nematology 17: 389-395.

O'Bannon, J.H. \& Nemec, S. 1979. The response of Citrus limon to a symbiot, Glomus etunicatus, and a pathogen, Radopholus similis. Journal of Nematology 11: 270-275.
PhILIPPS, J.M. \& HAYMAN, D.S. 1970. Improved procedures for clearing roots and staining parasitic and vesicular-arbuscular mycorrhizal fungi for rapid assessment of infection. Transactions of the British Mycological Society 55: 158-161.

SALEH, H. \& SiKorA, R.A. 1984. Relationship between Glomus fasciculatum root colonization of cotton and its effect on Meloidogyne incognita. Nematologica 30: 230-237.

SARAH, J.L. 1987. Utilisation d'une jachère travaillée pour lutter contre les nématodes parasites de l'ananas. Fruits 42: 357-360.

- 1991. Effect of soil pH on development of Pratylenchus brachyurus populations in pineapple roots. Nematropica 21: 211-216.

Sitaramaiah, K. \& Sikora, R.A. 1982. Effect of the mycorrhizal fungus Glomus fasciculatus on the host-parasite relationship of Rotylenchulus reniformis in tomato. Nematologica 28: 412-419.

SMITH, G.S. 1987. Interactions of nematodes with mycorrhizal fungi. In: Veech, J.A. \& Dickson, D.W. (eds.). Vistas on Nematology. Society of Nematologists, Hyattsville, MD. p. 292-300.

- \& KAPLAN, D.T. 1988. Influence of mycorrhizal fungus, phosphorus, and burrowing nematode interactions on growth of rough lemon citrus seedlings. Journal of Nematology 20: 539-544.

-, RONCADORI, R.W. \& HuSSEY, R.S. 1986. Interaction of endomycorrhizal fungi, superphosphate, and Meloidogyne incognita on cotton in microplot and field studies. Journal of Nematology 18: 208-216.

Smith, S.E. \& Gianinazzi-Pearson, V. 1990. Phosphate uptake and vesicular-arbuscular activity in mycorrhizal Allium cepa L.: effect of photon irradiance and phosphate nutrition. Australian Journal of Plant Physiology 17: 177-188.

Stirling, G.R. \& NikUlin, A. 1993. Population dynamics of plant parasitic nematodes in Queenland pineapple fields and the effects of these nematodes on pineapple production. Australian Journal of Experimental Agriculture 33: 197-206.

Suresh, C.K., BagyaraJ, D.J. \& Reddy, D.D.R. 1985. Effect of vesicular-arbuscular mycorrhiza on survival, penetration and development of root-knot nematode in tomato. Plant and Soil 87: 305-308.

Thomas, G.V., Sundararaju, P., Ali, S.S. \& Ghai, S.K. 1989. Individual and interactive effects of VA mycorrhizal fungi and root-knot nematode, Meloidogyne incognita, on cardamom. Tropical Agriculture 66: 21-24.

Tisserant, B., Gianinazzi-Pearson, V., Gianinazzi, S. \& Gollote, A. 1993. In planta histochemical staining of fungal alkaline phosphatase activity for analysis of efficient arbuscular endomycorrhizal infections. Mycological Research 97: 245-250.

Trouvelot, A., Kough, J. \& Gianinazzi-Pearson, V. 1986. Mesure du taux de mycorhization VA d'un système radiculaire. Recherche de méthodes d'estimation ayant une signification fonctionnelle. In: Gianinazzi-Pearson, 
V. \& Gianinazzi, S. (eds.). Mycorrhizae: Physiology and Genetics. INRA-Press, Paris. p. 217-221.

WARNER, M.H. \& Jones, J.B. 1967. Determination of total tissue using a Technicon Kjeldahl Nitrogen apparatus. Technicon Symposia 1966, Automation in analyti- cal chemistry, Vol. I. Mediad Inc., New York. p. 145148.

Manuscript received February 1994

\title{
SELOSTUS
}

\section{Arbuskelimykorritsasienten käyttö Pratylenchus brachyurus -ankeroisen torjunnassa mikrolisätyllä ananaksella}

\author{
Jean-Philippe Guillemin ', Silvio Gianinazzi', Vivienne Gianinazzi-Pearson' ja Jean Marchal ${ }^{2}$ \\ ' Laboratoire de Pytoparasitologie, INRA-CNRS, Station de Génétique et d'Ameloration des Plantes, INRA, Dijon, \\ Ranska ja ${ }^{2}$ Laboratoire de Physiologie et Biochimie, CIRAD, FLHOR, Montpellier, Ranska
}

\begin{abstract}
Ananaksen juuristosta tavattua ankeroista Pratylenchus brachyrus pidetään merkittävänä taudinaiheuttajana ananasviljelyksillä. Mikrolisättyihin ananaslajikkeisiin 'Queen Tahiti', 'Smooth Cayenne' ja 'Spanish' siirrostettiin Glomus-mykorritsasientä ja/tai ne tartutettiin P. brachyrus -ankeroisella. Siirrostus ja tartutus suoritettiin välittömästi ananaksen in vitro -vaiheen jälkeen tai kuukautta myöhemmin. Ankeroinen ei haitannut mykorritsallisten taimien kasvua. Ankeroisen aikainen tartutus heikensi mykorritsattomien taimien kasvua mutta myöhäinen tartutus
\end{abstract}

ei. Mykorritsan ansiosta ankeroisten lukumäärä/juurigramma väheni ankeroistartutuksen ajankohdasta riippumatta. Ankeroiset vähensivät merkittävästi mykorritsasienen kokonaisinfektiota lajikkeissa 'Queen Tahiti' ja 'Spanish' mutta eivät vaikuttaneet metabolisesti aktiivisten arbuskeleiden kehitykseen tutkittujen kolmen lajikkeen juuristossa. Mykorritsasiirrostus lisäsi kasvien versojen fosforipitoisuutta. Aikainen ankeroistartutus vähensi hiukan mykorritsattomien kasvien kivennäispitoisuuksia. 